\title{
ESTIMACIÓN DE LA TOXICIDAD DE BIOPRESERVANTES TÁNICOS SOBRE HONGOS XILÓFAGOS
}

\author{
Murace, Mónica ${ }^{1}$; Spavento, Eleana ${ }^{1}$; Luna, María Luján ${ }^{2}$ y Keil, Gabriel ${ }^{1}$
}

\section{RESUMEN}

Los tratamientos preservantes amplían las posibilidades de uso de las maderas a partir del aumento de su resistencia a la degradación fúngica (durabilidad) y, consecuentemente, su vida útil en situaciones para las que no presentan adecuada durabilidad natural. En términos generales, la preservación involucra la utilización de compuestos químicos potencialmente perjudiciales para el ser humano y para el medio ambiente. A causa de ello, la sustitución de dichos compuestos por extractivos provenientes de especies con alta durabilidad constituye una alternativa a considerar.

El objetivo del trabajo fue estimar la toxicidad de biopreservantes elaborados en base a extractos tánicos de quebracho colorado (Schinopsis balansae Engl.) sobre hongos xilófagos, mediante ensayos en laboratorio, aplicando la técnica de soil-block.

Se trabajó con madera de Slash Pine (Pinus elliottii Engelm.) constituida en su totalidad por albura, preservada por vació-presión (IRAM 9600, 1998) con soluciones hidrosolubles identificadas como Colatan IPG-A y Colatan IPG-B, elaboradas con extractos tánicos de quebracho colorado y reforzadas con sales minerales más un fijador de taninos.

En la madera impregnada fueron evaluadas la penetración y la retención de los preservantes, su distribución en el leño mediante microscopios estereoscópico y electrónico de barrido y la toxicidad de los mismos según norma IRAM 9518 (1962) en probetas expuestas a Pycnoporus sanguineus y Gloeophyllum sepiarium, hongos responsables de pudrición blanca y castaña, respectivamente.

La penetración de los preservantes en el leño fue total. Los valores de retención obtenidos fueron de $18,85 \mathrm{~kg} / \mathrm{m}^{3}$ y $19,40 \mathrm{~kg} / \mathrm{m}^{3}$ para IPG-A e IPG-B, respectivamente. Las soluciones se distribuyeron uniformemente en el tejido leñoso y se depositaron en las vías naturales de colonización fúngica. La madera tratada incrementó significativamente su resistencia a la degradación fúngica poniendo en evidencia el efecto tóxico de los productos ensayados. La mayor toxicidad se registró sobre la cepa de pudrición castaña, particularmente con el producto IPG-A. El tipo de penetración, los valores de retención y la distribución de las soluciones en el leño contribuyeron a explicar estos resultados.

En la madera de Pinus elliottii y ante las condiciones descritas, Colatan IPG-A e IPG-B resultan tóxicos para las cepas de ensayo y, en consecuencia, representan una alternativa natural de aumento de la resistencia a la degradación, en particular a la ocasionada por los hongos responsables de pudrición castaña.

Palabras Clave: Extractos tánicos, Schinopsis balansae, Pycnoporus sanguineus, Gloeophyllum sepiarium, degradación acelerada - soil-block.

\footnotetext{
1 Facultad de Ciencias Agrarias y Forestales, Universidad Nacional de La Plata. Calle 60 y 119 s/n, CP: 1900, CC 31, La Plata, Buenos Aires, Argentina. mmurace@gmail.com

2 Facultad de Ciencias Naturales y Museo, Universidad Nacional de La Plata, CIC-BA.
} 


\section{SUMMARY}

The preservative treatments expand the potential uses of timbers since they increase their resistance to fungal degradation (durability) and, as a consequence, their time of utility in situations for which they are not durable enough. Preservation involves mainly the use of chemical compounds which are potentially harmful for humans and the environment. Thus, the replacement of these compounds by extractives from long-lasting timbers constitutes an alternative to consider.

The aim of this work was to estimate the toxicity of biopreservatives developed on the basis of tannic extracts from Quebracho Colorado (Schinopsis balansae Engl.), on xylophagous fungi through laboratory tests, applying the soil block technique.

Pinus elliottii Engelm. samples (sapwood), vacuum-pressure preserved (IRAM 9600, 1998) with water-soluble solutions identified as Colatan IPG-A e IPG-B, developed from tannic extracts of Quebracho Colorado and reinforced with mineral salts and chemical bonding agents of tanins, were employed.

The penetration and retention of preservative substances were evaluated, as well as the distribution of solutions by means of stereoscopic and scanning electron microscopes. The toxicity of the preservatives was analyzed (IRAM 9518, 1962) in cubic blocks exposed to Pycnoporus sanguineus and Gloeophyllum sepiarium, white and brown rot fungi, respectively.

Both preservatives penetrated completely wood samples. The retention values were $18.85 \mathrm{~kg} / \mathrm{m}^{3}$ and $19.40 \mathrm{~kg} / \mathrm{m}^{3}$ for IPG-A e IPG-B, respectively. The solutions were uniformly distributed in the woody tissue and they were deposited in the natural ways of fungal colonization. Treated wood increased significantly its resistance to fungal degradation supporting the toxic effect of the tested products. Preservative substances resulted more toxic for the brown rot strain, in particular the product IPG-A. The complete penetration of solutions in wood samples, as well as their retention values and homogenous distribution in the tissue contributed to explain the results.

Under described conditions and employing Pinus elliottii Engelm. wood samples, Colatan IPG-A and IPG-B are toxic for the tested strains and, as consequence, they represent a natural alternative to increase wood resistance to degradation, particulary those caused by brown rot fungi.

Key Words: Tannic extracts, Schinopsis balansae, Pycnoporus sanguineus, Gloeophyllum sepiarium, accelerated degradation, soil-block. 


\section{INTRODUCCIÓN}

En la madera en servicio, los hongos xilófagos son considerados los agentes de deterioro más agresivos, debido a que producen su alteración estructural (pudrición), en períodos de tiempo relativamente cortos; el hábito de crecimiento miceliar y la actividad enzimática de estos organismos determinan su carácter de eficientes colonizadores y transformadores del sustrato.

La pudrición de la madera resulta de la degradación ectoenzimática de los componentes principales de la pared celular; celulosa, hemicelulosas y lignina. La rápida despolimerización de estos componentes desde los estadios iniciales del proceso de degradación (pérdida de peso $\leq 10$ \%) determina en la madera cambios químicos y estructurales que conducen a la reducción temprana de sus propiedades de resistencia, limitando consecuentemente su vida útil en situaciones propicias para este tipo de deterioro (contenidos de humedad superiores al punto de saturación de las fibras: 25\%) (Wilcox, 1978; Zabel y Morrell, 1992; Ah Chee et al., 1998; Curling et al., 2002; Barnet y Jeronimides, 2003; Archer y Lebow, 2006).

La resistencia natural de las maderas a la degradación fúngica (durabilidad natural) puede ser incrementada mediante tratamientos preservantes (durabilidad adquirida). De acuerdo con esto, las prácticas de preservación amplían las posibilidades de uso de las maderas a partir del aumento de su vida útil en situaciones para las que no presentan adecuada aptitud natural (Diaz et al., 2003; Miller et al., 2003; Schmidt, 2006; Alves da Silva et al., 2007; Lebow, 2010).

En términos generales, la preservación involucra el uso de compuestos químicos potencialmente perjudiciales para el ser humano y para el medio ambiente. A causa de ello, el uso de extractivos naturales (biopreservantes) es considerado y estudiado como una alternativa viable (Rodríguez Barreal, 1998; Onuorah, 2000; Kumar y Gupta, 2006; Velásquez et al., 2006; Bernardis y Popoff, 2009). Da Silva Oliveira et al. (2000) y Thévenon et al. (2010) mencionan el tratamiento de las maderas no durables con extractivos provenientes de especies con alta durabilidad natural como también la utilización de dichos compuestos como modelos de síntesis de nuevos preservantes.

Entre los extractivos del duramen, los taninos son considerados como preservantes naturales dada su fuerte influencia en el retraso de la colonización y degradación fúngica y consecuentemente, en el aumento de la durabilidad de las maderas. Los efectos inhibitorios de estos compuestos aromáticos sobre el crecimiento miceliar y las enzimas de los hongos son relacionados con la formación de productos complejos resultantes de su asociación con distintas biomoléculas - proteínas, almidón, sustancias pécticas, celulosa -. Asimismo es reconocida la resistencia al biodeterioro fúngico que se obtiene con la formación de complejos insolubles constituidos por sales minerales y taninos (Hillis, 1968; Hart y Hillis, 1972; Blanchette y Biggs, 1992; Zabel y Morrell, 1992; Gonzáles Laredo, 1996; Schwarze et al., 2000; Aloui et al., 2004; Barnet y Jeronimidis, 2003; Velásquez et al., 2006; Lekounougou et al., 2008; Thévenon et al., 2010).

Ante la necesidad de analizar los efectos de nuevos principios activos y/o formulaciones químicas sobre la acción de los hongos xilófagos, los ensayos de laboratorio constituyen una alternativa viable y ventajosa debido a los cortos periodos de tiempo en los que se obtienen resultados. En la República Argentina, la Norma IRAM 9518 (1962) propone una metodología que permite evaluar la toxicidad, permanencia y eficacia de productos preservantes.

En cuanto a la toxicidad, ésta es estimada mediante la determinación del porcentaje medio de pérdida de peso en probetas preservadas y expuestas a la acción de cepas xilófagas a través de ensayos de laboratorio - ensayos de biodegradación acelerada - aplicando la técnica del soil-block. A los fines prácticos, la estimación de la toxicidad resulta adecuada solo para aquellos productos que se apliquen a maderas de uso interior y sin contacto con el suelo. 
Como hipótesis de trabajo se considera que los biopreservantes tánicos son tóxicos para los hongos xilófagos $y$, en consecuencia, incrementan la resistencia de las maderas a la degradación fúngica.

\section{OBJETIVO}

Estimar la toxicidad de biopreservantes elaborados en base a extractos tánicos de quebracho colorado (Schinopsis balansae Engl.) sobre hongos xilófagos, mediante ensayos de degradación en laboratorio, aplicando la técnica de soil-block.

\section{MATERIALES Y MÉTODOS}

\section{Material Leñoso}

El material consistió en madera de Pinus elliottii Engelm. proveniente de aserraderos de la localidad de El Dorado ( $\left.26^{\circ} 25^{\prime} \mathrm{S}, 54^{\circ} 37^{\prime} \mathrm{W}\right)$, provincia de Misiones, Argentina.

Se trabajó con listones constituidos en su totalidad por albura, secos y cepillados, de sección cuadrangular, de $20 \mathrm{~mm}$ de lado y $1200 \mathrm{~mm}$ de longitud. Para cada listón fue determinado el contenido de humedad (CH\%) mediante el método de pesadas (IRAM 9532, 1963) y su volumen (Va) con calibre digital (medición directa o estereometría).

\section{Impregnación del Material Leñoso}

\section{- Sustancias Preservantes}

Los preservantes utilizados correspondieron a la marca comercial Colatan IPG perteneciente a la Empresa UNITAN SAICA: Colatan IPG-A y Colatan IPG-B.

Dichos productos consisten en soluciones hidrosolubles elaboradas con extractos tánicos de quebracho colorado, reforzadas con sales minerales más un fijador de tanino para evitar su lavado. La concentración (C) utilizada fue del $4 \%$.

\section{- Método de Impregnación}

El material de ensayo fue impregnado por vacío - presión (VP) mediante el método Bethell - célula llena tradicional - (IRAM 9600, 1998). Los parámetros empleados en la impregnación por VP son especificados en el Cuadro $N^{\circ} 1$. Fue impregnado un total de 6 listones, los cuales presentaron un $\mathrm{CH}$ promedio del $15,5 \%$, valor aceptable según la normativa indicada.

\section{PARÁMETROS EMPLEADOS EN LA IMPREGNACIÓN POR VP}

\begin{tabular}{|l|c|}
\hline Parámetro de Impregnación & Valores Reales de Trabajo \\
\hline Concentración de la solución (\%) & 4 \\
Vacío inicial: magnitud (Mg)* & 600 \\
Vacío inicial: duración (minutos) & 15 \\
Presión: magnitud (Kg/cm²) & 5 \\
Presión: duración (minutos) & 20 \\
Vacío final: magnitud (Mg.) $^{*}$ & 600 \\
Vacío final: duración (minutos) $^{*}$ & 5 \\
\hline
\end{tabular}

* Las unidades de vacío y presión son las de uso común en la industria. 
La impregnación fue realizada en la planta piloto de la Facultad de Ciencias Agrarias y Forestales, Universidad Nacional de La Plata (UNLP), Argentina.

\section{- Penetración y Cálculo de la Retención de los Preservantes}

La penetración de los preservantes fue estimada a ojo desnudo, a partir de la visualización de la profundidad alcanzada por el teñido que generaron las soluciones preservantes.

En cuanto al cálculo de la retención $(\mathrm{R})$, los listones de madera fueron pesados antes (Pi) y después (Pf) de la impregnación. Luego, considerando la concentración (C) empleada y el Va, fueron obtenidos los valores de $\mathrm{R}$ mediante la siguiente fórmula:

$$
R\left(\mathrm{Kg} / \mathrm{m}^{3}\right)=\frac{\mathrm{Pf}(\mathrm{Kg})-\mathrm{Pi}(\mathrm{Kg})}{\mathrm{Va}\left(\mathrm{m}^{3}\right)} \times \frac{\mathrm{C}(\%)}{100}
$$

Culminada esta etapa, del total de listones impregnados fueron obtenidas 90 probetas cúbicas de $20 \mathrm{~mm}$ de lado, libres de defectos (45 por cada producto ensayado) con el propósito de evaluar la toxicidad de los biopreservantes tánicos mediante la técnica del soil block así como su distribución en la madera a partir de los estudios anatómicos.

\section{Análisis Anatómico}

Los estudios anatómicos fueron realizados sobre un total de 10 probetas impregnadas ( 5 por sustancia preservante). El material fue observado con lupa y con microscopio electrónico de barrido (MEB), perteneciente al Servicio de Microscopía Electrónica de la Facultad de Ciencias Naturales y Museo (UNLP), Argentina.

Para las observaciones con MEB, se realizaron cortes transversales y longitudinales de madera obtenidos con cuchillas de metal, los cuales fueron montados sobre los tacos sin tratamiento previo y metalizados con un baño de oro-paladio.

\section{Biodegradación}

\section{- Material Fúngico}

Las cepas xilófagas utilizadas fueron Pycnoporus sanguineus (L. ex Fr.) Bull. Murr. cepa 163 (LPSC) y Gloeophyllum sepiarium (Wulf.: Fr.) P. Karst. cepa 735 (LPSC) - Cepario del Instituto Spegazzini, La Plata, causantes de pudrición blanca $(\mathrm{Pb})$ y castaña $(\mathrm{Pc})$, respectivamente, consideradas especies de referencia para este tipo de ensayos (IRAM 9518, 1962; Mora y Encinas, 2001; Bobadilla et al., 2005; Alves da Silva et al., 2007; Bernardis y Popoff, 2009, Thévenon et al., 2010).

El micelio fúngico fue repicado en cajas de Petri con medio de cultivo agar extracto de malta $(\mathrm{AEM})$ e incubado en estufa a $25^{\circ} \mathrm{C} \pm 2$ durante 15 días.

\section{-Material Leñoso}

Los ensayos de biodegradación fueron realizados con probetas cúbicas de albura de pino preservadas y sin tratamiento (testigos), de $20 \mathrm{~mm}$ de lado, libres de defectos y $\mathrm{CH}$ del $15,5 \%$. 
Se trabajó con un total de 120 probetas, 80 impregnadas y 40 testigos (20 por condición de la madera; madera testigo y madera preservada con cada uno de los preservantes vs cepa xilófaga).

\section{Instalación de los Ensayos}

Los ensayos de biodegradación fueron realizados en laboratorio según norma IRAM 9518 (1962). En frascos de vidrio de $500 \mathrm{~cm}^{3}$ de capacidad fueron colocados $43 \mathrm{~cm}^{3}$ de agua destilada y $200 \mathrm{~g}$ de una mezcla de tierra $(40 \mathrm{~g})$ y arena $(160 \mathrm{~g})$, previamente secada en estufa a $103 \stackrel{\circ}{\circ} \mathrm{C} \pm 2$ durante $72 \mathrm{~h}$ y posteriormente tamizada.

Llenados los frascos, sobre el sustrato fueron apoyadas dos tablillas (feeder strips) de madera blanda (Salix spp) de $20 \mathrm{~mm}$ de lado y $4 \mathrm{~mm}$ de espesor. Posteriormente, cada frasco fue tapado con tapones de algodón y esterilizado en autoclave durante 30 minutos a 1,5 atm de presión.

Cumplida esta etapa se procedió a la inoculación, la cual fue realizada mediante trozos de micelio fúngico de $1 \mathrm{~cm}$ de diámetro colocados próximos a cada feeder strips.

Una vez que el micelio cubrió las tablillas (20 días), sobre cada una de ellas fue colocada una probeta de ensayo (dos por frasco), previa determinación de su peso inicial en equilibrio higroscópico $(\mathrm{Pi})$ y esterilización en autoclave a $1 \frac{1}{2}$ atm de presión durante 20 minutos.

El material estuvo expuesto a degradación durante 90 días a $27 \pm 2{ }^{\circ} \mathrm{C}$ y $70 \%$ de HR. Cumplido el tiempo de ensayo, las probetas fueron extraídas de los frascos y acondicionadas en laboratorio (30 días aproximadamente) bajo condiciones controladas de temperatura y humedad $(27 \pm 2 \stackrel{\circ}{\circ}$ y $70 \%$ de HR) hasta comprobar peso en equilibrio higroscópico (Pf). Con los $\mathrm{Pi}$ y $\mathrm{Pf}$ obtenidos fue calculada la media de pérdida de peso (Pp\%) para cada condición de la madera vs cepa xilófaga empleando la fórmula 2:

$$
P p(\%)=\frac{P i(g)-P f(g)}{P i(g)} \times 100
$$

Determinada la $\mathrm{Pp}(\%)$, los datos obtenidos permitieron determinar la toxicidad de los preservantes sobre las cepas xilófagas mediante el cálculo de diferencias en el porcentaje medio de $\mathrm{Pp}$ entre el material preservado con cada una de las sustancias y el testigo, como así también evaluar la toxicidad diferencial entre cepas por producto.

Estos ensayos fueron realizados en el Laboratorio de Protección Forestal, Facultad de Ciencias Agrarias y Forestales (UNLP), Argentina.

\section{Diseño Experimental - Análisis Estadístico}

Como diseño experimental se adoptó el completamente aleatorizado, con análisis factorial 3 por 2; siendo 3 las condiciones de las probetas testigo y tratadas con los preservantes Colatan IPG-A e IPG-B y 2 las cepas xilófagas o tipos de pudrición: pudrición blanca $\mathrm{Pb}: P$. sanguineus y pudrición castaña Pc: G. sepiarium.

La variable medida fue el peso de cada una de las probetas al inicio (Pi) y al final del ensayo (Pf). Con estos valores fue obtenido el porcentaje medio de pérdida de peso (Pp) para cada combinación: condición de la madera - tipo de pudrición. 
Obtenidas las medias de Pp (\%), éstas fueron expuestas al análisis de la varianza (ANOVA) y test de Tukey (diferencias significativas $p<0.05$ ) mediante el programa Infostat. Del mismo modo fueron analizados los valores medios de $R$ obtenidos para cada sustancia preservante.

\section{RESULTADOS Y DISCUSIÓN}

\section{Penetración y Valores de Retención Obtenidos}

En los listones de Pinus elliotti impregnados se observó la penetración total del Colatán IPG-A y del Colatán IPG-B. Los valores medios de retención (R) son presentados en el Cuadro $\mathrm{N}^{\circ}$ 2.

Los resultados obtenidos fueron los esperados para esta madera constituida en su totalidad por albura impregnada mediante VP y considerando la importancia de los valores de estos parámetros al momento de analizar la calidad y posible efectividad del tratamiento utilizado.

En las maderas tratadas, el grado de protección contra el deterioro depende del preservante usado como también de su tipo de penetración, de su distribución y de su retención, aspectos estrechamente vinculados con el método de impregnación y con la naturaleza del tejido leñoso (Lebow, 2010).

Bernardis y Popoff (2009) obtuvieron retenciones de 9,18 y $25 \mathrm{~kg} / \mathrm{m}^{3}$ para la madera de esta especie impregnada por el mismo método y con productos de composición química similar, con valores ligeramente distintos en duración y magnitud de la presión empleada.

\section{Análisis Anatómico}

Con microscopio binocular (lupa) se observó la distribución uniforme del Colatán IPG-A y del IPG-B en el tejido leñoso. Con MEB se determinó que los biopreservantes fluyeron por las vías naturales de traslado de líquidos en las maderas de coníferas.

De acuerdo con esto, fueron observados depósitos de estas sustancias en el lumen de las células parenquimáticas de los radios, de las traqueidas y de las fibrotraqueidas, y sobre la pared celular, impregnando inclusive las punteaduras de las células. Asimismo, fueron visualizados depósitos en el interior de los canales resiníferos axiales los cuales no se hallaban obliterados por resinas.

La distribución de los preservantes (tipo y ubicación) fue la esperada para la albura de coníferas impregnada por VP, lo cual contribuiría a su resistencia a la colonización y degradación fúngica.

Como se mencionó, en las maderas tratadas el grado de protección contra el deterioro depende, entre otros, del tipo de distribución de la sustancia preservante, característica estrechamente vinculada con el método de impregnación y con la naturaleza del tejido leñoso (Lebow, 2010).

En las maderas, las vías de colonización fúngica las constituyen los diferentes tipos celulares del sistema axial (vasos, traqueidas y fibras) y su alineamiento, como así también la organización radial del parénquima.

El movimiento a través de las punteaduras o la penetración directa de la pared celular mediante perforaciones producidas por los hongos (bore holes) son vías de colonización de los hongos en la madera (Schwarze, 2007), que según lo hallado en este trabajo fueron interrumpidas por la presencia de los biopreservantes. 

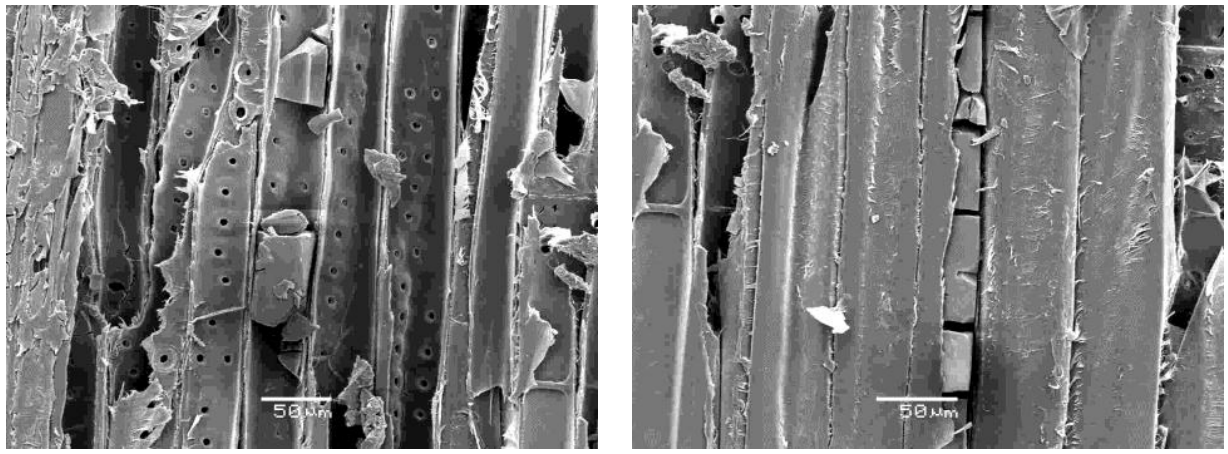

Depósitos de preservante en traqueidas

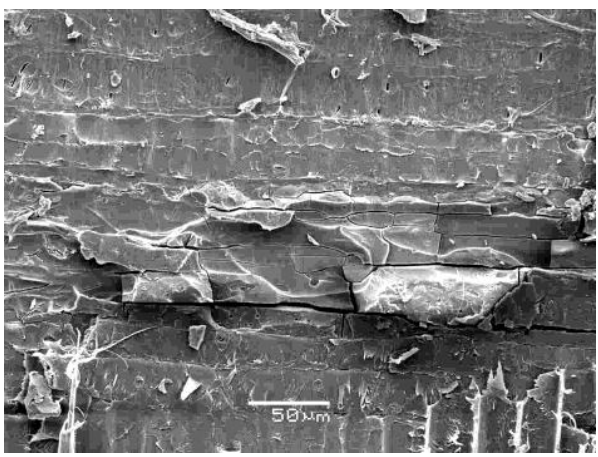

Depósitos de preservante en fibrotraqueidas

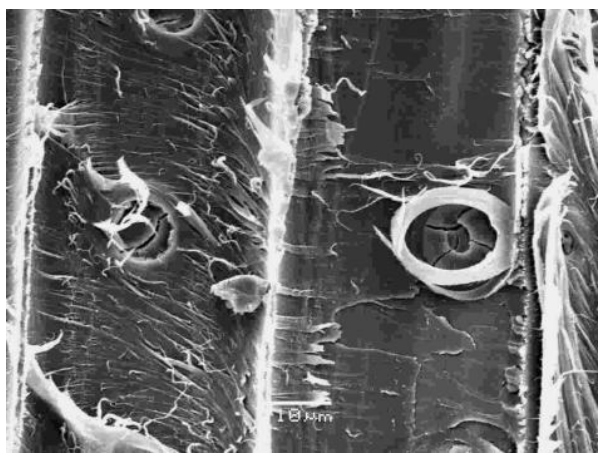

Depósitos de preservante en radios

Depósitos de preservante en punteaduras

Figura № 1

DISTRIBUCIÓN DEL PRESERVANTE IPG-A EN EL LEÑO DE Pinus elliotii

\section{Biodegradación}

Los porcentajes medios de $\mathrm{Pp}$ estimados para las probetas testigos y las tratadas con Colatán IPG-A y Colatán IPG-B, expuestas a $P$. sanguineus y $G$. sepiarium, se indican en el Cuadro $\mathrm{N}^{\circ} 2$.

De acuerdo a lo esperado, los porcentajes medios de Pp más altos fueron obtenidos para el material testigo (Cuadro $\mathrm{N}^{\circ} 2$ ). Bernardis y Popoff (2009) arribaron a resultados semejantes con las mismas especies xilófagas y sustancias preservantes similares.

Para esta condición de la madera, el porcentaje medio de Pp significativamente superior determinado en el material expuesto a la cepa de pudrición castaña coincide con estudios previos, en los que se pone en evidencia la alta susceptibilidad de las Gimnospermas a este tipo de pudrición (Winandy y Morrell, 1993; Morrell y Freitag, 1995; Juacida y Villanueva, 1996; Mora y Encinas, 2001; Curling et al., 2002; Miller et al., 2003; Pandey y Pitman, 2003; Schwarze et al., 2003, Bernardis y Popoff, 2009).

Schwarze (2007) menciona la alta adaptación de estos hongos al leño relativamente simple de las Gimnospermas. La menor Pp (\%) registrada con la cepa de pudrición blanca estaría 
asociada al tipo de lignina presente en la madera ensayada. La lignina de las coníferas contiene unidades guayacil - propil caracterizadas por su resistencia a la degradación por este tipo de hongos xilófagos (Blanchette, 1995; Reid, 1995; Curling et al., 2002; Martínez et al., 2005; Schwarze, 2007).

\section{Cuadro $\mathrm{N}^{\circ} 2$}

PORCENTAJES MEDIOS DE PÉRDIDA DE PESO OBTENIDOS POR CONDICIÓN DE LA MADERA VS CEPAS XILÓFAGAS

\begin{tabular}{|c|c|c|}
\hline \multirow{2}{*}{$\begin{array}{l}\text { Condición de la Madera / R } \\
\qquad\left(\mathrm{Kg} / \mathrm{m}^{3}\right)\end{array}$} & \multicolumn{2}{|c|}{$\begin{array}{c}\text { Pérdida de Peso } \\
(\%)^{*}\end{array}$} \\
\hline & $\begin{array}{c}\text { P. sanguineus } \\
\mathrm{Pb}\end{array}$ & $\begin{array}{c}\text { G. sepiarium } \\
\text { Pc }\end{array}$ \\
\hline Madera sin impregnar (Testigo) & $\begin{array}{c}25,25 \text { a A } \\
(7,46)\end{array}$ & $\begin{array}{c}36,76 \text { a B } \\
(12,28)\end{array}$ \\
\hline $\begin{array}{c}\text { IPG - B a } \\
19,40 \mathrm{Kg} / \mathrm{m}^{3}\end{array}$ & $\begin{array}{c}19,38 \mathrm{~b} A \\
(11,79)\end{array}$ & $\begin{array}{c}17,84 \mathrm{~b} A \\
(13,80)\end{array}$ \\
\hline $\begin{array}{c}\text { IPG - A a } \\
18,85 \mathrm{Kg} / \mathrm{m}^{3}\end{array}$ & $\begin{array}{c}12,57 \mathrm{c} \mathrm{A} \\
(17,12)\end{array}$ & $\begin{array}{c}4,99 \text { c B } \\
(30,21)\end{array}$ \\
\hline
\end{tabular}

* Letras distintas denotan diferencias estadísticamente significativas al 95\% de confianza en sentido vertical (minúsculas) y horizontal (mayúsculas). El coeficiente de variabilidad (CV\%) se muestra entre paréntesis. R: retención. Pb: pudrición blanca. Pc: pudrición castaña.

En referencia a la madera tratada, su mayor resistencia a la degradación fúngica (Cuadro $\mathrm{N}^{\circ}$ 2) puso de manifiesto la toxicidad de los biopreservantes IPG-A e IPG-B, resultados esperados teniendo en cuenta la $\mathrm{P}$, distribución y los valores de $\mathrm{R}$ obtenidos, como así también la naturaleza y origen de estas sustancias.

Roth y Giménez de Bolzón (1997) relacionan la alta durabilidad natural de las especies de Schinopsis con la elevada proporción de taninos (próxima al 65\%) que posee su duramen. Borlando (1953) y Miller et al. (2003) mencionan la alta resistencia a la degradación fúngica de diferentes especies de este género botánico.

Los preservantes resultaron más tóxicos para la cepa G. sepiarium, particularmente el denominado IPG-A (Cuadro $N^{\circ}$ 2). Bernadis y Popoff (2009) obtuvieron iguales resultados para el tratamiento Pinus elliotti vs G. sepiarium utilizando un producto de naturaleza similar (IPG-C) con valores de $R$ de 9 y $25 \mathrm{~kg} / \mathrm{m}^{3}$.

Velásquez et al. (2006) determinaron el mayor efecto inhibidor de los extractivos del duramen de latifoliadas sobre $G$. trabeum, especie causante de pudrición castaña, en relación a sus efectos sobre Trametes versicolor, agente de pudrición blanca.

Hart y Hillis (1972) al estudiar el efecto de los elagitaninos, taninos hidrolizables, sobre hongos responsables de pudriciones blanca y castaña, determinaron una mayor inhibición del poder degradativo y del crecimiento sobre la cepa de pudrición castaña Poria monticola.

Existen antecedentes acerca de la fuerte inhibición del catecol, flavonoide que por polimerización origina los taninos condensados, sobre las xilanasas y $\beta$-1,4-endoglucanasas del hongo de pudrición castaña Postia placenta (Highley y Micales, 1990, tomado de Highley y Illman, 1991; Kumar y Gupta, 2006). Los hongos de pudrición blanca y de pudrición castaña poseen enzimas diferentes en cuanto a tamaño molecular y modo de acción, características determinantes 
en la formación de complejos taninos - proteínas, forma bajo la cual los compuestos aromáticos ejercen sus efectos inhibitorios (Hart y Hillis, 1972; González Laredo, 1996).

Por su parte, los hongos de pudrición blanca poseen un potente sistema enzimático extracelular oxidativo conformado por peroxidasas y lacasas, sistema ligninolítico, que les permite la degradación de numerosos compuestos orgánicos, entre ellos fenoles y polifenoles, y por esto considerados como potenciales agentes detoxificadores. En relación a esto, existen antecedentes acerca de la oxidación de taninos condensados, catequinas polifenólicas, catalizada por lacasas (Reid, 1995; Saparrat et al., 2002; Lekounougou et al., 2008; Rubilar et al., 2008). Lo expuesto aquí permitiría explicar la resistencia diferencial a la degradación de la madera preservada en particular con IPG-A (Cuadro N²).

En referencia a $P$. sanguineus, su menor capacidad degradativa sobre la madera tratada en relación al material testigo (Cuadro $\mathrm{N}^{\circ} 2$ ), sería consecuencia del efecto de los biopreservantes sobre su desarrollo miceliar y actividad enzimática (Hart y Hillis, 1972). Taylor et al. (1987), tomado de Kumar y Gupta (2006), mencionan la disminución del crecimiento de T. versicolor ante la presencia de catecol. Highley y Micales (1990), tomado de Kumar y Gupta (2006), comprobaron los efectos inhibitorios de compuestos aromáticos monoméricos sobre el crecimiento de dicha cepa xilófaga.

En oposición a lo hallado en la presente investigación, Bernardis y Popoff (2009) determinaron una mayor inhibición de la capacidad degradativa de $P$. sanguineus (Pp entre 3,5 y 8 $\%$ ) sobre la misma madera tratada con biopreservantes semejantes (IPG-C e IPG-F), con valores de $\mathrm{R}$ entre 9 y $25 \mathrm{~kg} / \mathrm{m}^{3}$. Estos resultados opuestos podrían deberse a diferencias en la concentración de los constituyentes de la formulación o bien a diferencias existentes en torno a la naturaleza química de las sales minerales utilizadas como refuerzo de los extractos tánicos, factores determinantes de condiciones microambientales diferentes y del impacto en los patrones de crecimiento y de producción enzimática fúngica (Lekounougou et al., 2008, Rubilar et al., 2008). Del mismo modo podrían explicarse las diferencias significativas en torno al porcentaje medio de Pp encontrado entre las maderas impregnadas con los biopreservantes IPG-A e IPG-B expuestas a $P$. sanguineus y $G$. sepiarium (Cuadro $N^{\circ} 2$ ).

Según lo hallado en el presente estudio, las maderas tratadas con estos productos verían incrementada su vida útil en servicio, particularmente con Colatán IPG-A, en condiciones de uso interior, sin contacto con el suelo y expuestas a situaciones propicias para este tipo de deterioro (elevado contenido de humedad producto de defectos constructivos, ambientes confinados, poco ventilados, entre otros).

Posteriores investigaciones deberían conducirse a los efectos de analizar el comportamiento de estas soluciones en maderas expuestas a ciclos de intemperismo (estimación de la permanencia, capacidad de fijación de los preservantes en la madera), como también su eficacia (relación entre retención límite de permanencia y retención límite tóxica), con el objeto de estimar las potencialidades de estos biopreservantes en situaciones extremas de uso (madera al exterior y en contacto con el suelo).

No obstante los resultados obtenidos para los distintos tratamientos (condición de la madera vs cepa xilófaga), debe tenerse en cuenta que las pudriciones castañas son consideradas las más agresivas, ya que causan una rápida e intensa despolimerización de la holocelulosa desde los estadios iniciales del proceso de degradación, mientras que la lignina solo es parcialmente oxidada y responsable de la coloración castaña característica que adquiere la madera podrida.

Esta particularidad en cuanto a la degradación de los carbohidratos conduce a una abrupta disminución de la capacidad de resistencia de la madera (principalmente atribuida a la despolimerización de las hemicelulosas y no proporcional con la pérdida de peso) y, en consecuencia, a una notable reducción de su vida útil en servicio (Wilcox, 1978; Highley e Illman, 
1991; Highley 1987a, tomado de Green III y Highley, 1997; Green III et al., 1991; Winandy y Morrell, 1993; Schwarze et al., 2000; Schwarze, 2007; Emerhi et al., 2008; Murace et al., 2010).

\section{CONCLUSIONES}

En la madera de Pinus elliottii Engelm. y ante las condiciones descritas (leño conformado por albura, concentración de los preservantes, método de preservación, tipo de penetración, valor de retención, distribución homogénea de los productos en el tejido y su ubicación en las vías de colonización fúngica) los biopreservantes Colatan IPG-A y Colatan IPG-B elaborados en base a extractos tánicos del quebracho colorado (Schinopsis balansae) resultan tóxicos para Pycnoporus sanguineus y Gloeophyllum sepiarium, cepas responsables de pudrición blanca y castaña, respectivamente.

Ambos productos son más efectivos sobre la cepa de pudrición castaña con respecto al material testigo, en particular el IPG-A. Asimismo, para ambas cepas xilófagas, Colatan IPG-A produce el mayor efecto inhibidor de la degradación y con diferencias significativas entre tipo de pudrición.

De acuerdo con esto, los preservantes, y particularmente el IPG A, representan una alternativa natural de aumento de la resistencia a la degradación fúngica para las maderas con uso interior, sin contacto con el suelo y expuestas a situaciones propicias para este tipo de deterioro, principalmente el ocasionado por los hongos responsables de pudrición castaña.

\section{REFERENCIAS}

Ah Chee, A.; Farrell, R. L.; Stewart, A. y Hill, R. A., 1998. Decay potential of basidiomycete fungi from Pinus radiata. Proc. 51 st. N. Z. Plant Protection Conf. pag. 235-240.

Aloui, F.; Ayadi, N.; Charrier, F. y Charrier, B., 2004. Durability of European Oak (Quercus petraea and Quercus robur) against white rot fungi (Coriolus versicolor): relations with phenol extractives. Holz Roh Werkst 62:286-290.

Alves da Silva, C.; Monteiro, M. B. B.; Brazolin, S.; López, G. A. C.; Richter, A. y Braga, M. R., 2007. Biodeteriotation of Brazilwood Caesalpinia echinata Lam. (Leguminosa-Caesalpinioideae) by rot fungi and termites. International Biodeteriotation \& Biodegradation 60:285-292.

Archer, K. y Lebow, S., 2006. Wood preservation. In: Primary wood processing: Principles and practice. $2^{\text {nd }}$ Edition. Springer, The Netherlands, pag.: 297-338.

Barnett, J. R. y Jeronimidis, G., 2003. Wood quality and its biological basis. Blackwell Publishing Ltd. CRC Press. $240 \mathrm{pp}$.

Bernardis, A. C. y Popoff, O., 2009. Durability of Pinus elliottii wood impregnated with Quebracho Colorado (Schinopsis balansae) bio-protectives extracts and CCA. Maderas, Ciencia y Tecnología, 11(2): 107-115.

Blanchette, R., 1995. Degradation of the lignocellulose complex in wood. Can. J. Bot. 73(Suppl.1): 999-1010.

Blanchette, R. A. y Biggs, A. R., 1992. Defense mechanisms of woody plants against fungi. Springer Series in Wood Science. Editor, T. E. Timell. Cap. 8: Biochemistry of Gymnosperm xylem responses to fungal invasion 147164 by T. Yamada.

Bobadilla, E. A.; Pereyra, O.; Silva, F. y Stehr, A. M., 2005. Durabilidad naural de la madera de dos especies aptas para la industria de la construcción. Floresta 35 (3): 419-428.

Borlando, I., 1953. Determinación de la durabilidad natural de algunas maderas argentinas. Ministerio de Obras Públicas. Laboratorio de Ensayo de Materiales e Investigaciones Tecnológicas. Serie II, Número 51. pp: 5-23. 
Curling, S. F.; Clausen, C. A. y Winandy, J. E., 2002. Experimental method to quantify progressive stages of decay of wood by basidiomycete fungi. International Biodeterioration \& Biodegradation 49: 13-19.

Da Silva Oliveira, J. T.; Hellmeister, J. C. y Filho, M. T., 2000. Eucalypt wood characterization for construction in Brazil - Natural durability. I Congreso Iberoamericano de Investigación y Desarrollo de Productos Forestales.

Díaz, B.; Murace, M.; Peri, P.; Keil, G; Luna, M. L. y Otaño, M., 2003. Natural and preservative treated durability of Populus nigra cv Italica timber grown in Santa Cruz Province, Argentina. International Biodeterioration \& Biodegradation. 52 (2003): 43 - 47.

Emerhi, E. A.; Ekeke, B. A. y Oyebade, B. A., 2008. Biodegrading effects of some rot fungi on Pinus caribaea wood. African Journal of Biotechnology. Vol 7(10): 1512-1515.

Green III, F. y Highley, T. L., 1997. Mechanism of Brown-rot decay: paradigm or paradox. International Biodeterioration \& Biodegradation. Vol. 39. №2-3: 113-124.

Green III, F.; Larsen, M. J.; Winandy, J. E. y Highley, T. L., 1991. Role of oxalic acid in incipient brown - rot decay. Material und Organismen 26. Bd. Heft 3. Verlag Duncker \& Humblot. 1000 Berlin 41. Pág. 191-213.

González Laredo, R. F., 1996. Preservación de madera con taninos. Madera y Bosques 2(2):67-73.

Hart, J. H. y Hillis, E. E., 1972. Inhibition of wood-rotting fungi by ellagitannins in the heartwood of Quercus alba. Phytopathology 62: 620-626.

Highley, T. L. y Illman, B. L., 1991. Progress in understanding how brown-rot fungi degrade cellulose. Biodeterioration Abstracts. 5(3): 231-244.

Hillis, E. E., 1968. Chemical aspects of heartwood formation. Wood Science \& Technology 2:241-259.

IRAM 9518, 1962. Toxicidad, Permanencia y Eficacia de Preservadores de Madera. Instituto Argentino de Racionalización de Materiales. 12 pp.

IRAM 9532, 1963. Método de determinación de humedad. Instituto Argentino de Racionalización de Materiales. $14 \mathrm{pp}$.

IRAM 9600, 1998. Preservación de maderas. Maderas preservadas mediante procesos con presión en autoclave. Instituto Argentino de Racionalización de Materiales. 23 pp.

Juacida, R. y Villanueva, J., 1996. Durabilidad natural de Sequoia sempervirens (D. Don) Endl. Bosque 17(1): 83-90.

Kumar, D. y Gupta, R. K., 2006. Biocontrol of wood-rotting fungi. Indian Journal of Biotechnology. Vol. 5, January, 20-25.

Lebow, S. T., 2010. Wood preservation In: Wood handbook - Wood as an engineering material: U.S. Department of Agriculture Forest Service. Forest Products Laboratory. General Technical Report FPL-GTR-190. Madison, Wisconsin, pag.: 15-1 a 15-28.

Lekounougou, S.; Mounguengui, S.; Dumarcay, S.; Rose, C.; Courty, P. E.; Garbaye, J.; Gerardin, P.; Jacquot, J. P. y Gelhaye, E., 2008. Initial stages of Fagus sylvatica wood colonization by the white-rot basidiomycete Trametes versicolor: Enzymatic characterization. International Biodeterioration \& Biodegradation 61: $287-293$.

Martínez, A. T.; Speranza, M.; Ruiz-Dueñas, F. J.; Ferreira, P.; Camarero, S.; Guillén, F.; Martínez, M. J.; Gutiérrez, A. y del Río, J. C., 2005. Biodegradation of lignocellulosics: microbiol, chemicals, and enzimática aspects of the fungal attack of lignin. International Microbiology 8: 195-204.

Miller, R. B.; Wiedenhoeft, A. C.; Williams, R. S.; Stockman, W. Y Green III, F., 2003. Characteristics of ten tropical hardwoods from certified forests in Bolivia. Part II natural durability to decay fungi. Wood and Fiber Science. 35(3): 429-433.

Mora, N. y Encinas, O., 2001. Evaluación de la durabilidad natural e inducida de Pterocarpus acapulcensis, Tabebuia serratifolia y Pinus caribaea en condiciones de laboratorio. Rev. Forest. Venez. 45(1): 23-31. 
Morrell, J. J. y Freitag, C. M., 1995. Durability of Dahurian Larch. Forest products Journal. Vol. 45(1): 77-78.

Murace, M.; Spavento, E.; Keil, G. D. y Saparrat, M., 2010. Pudrición castaña: Efectos sobre las propiedades de resistencia mecánica de la madera. Quebracho - Revista de Ciencias Forestales 18(1,2): 37-46.

Onuorah, E. O., 2000. The wood preservative potentials of heartwood extracts of Milicia excelsa and Erythrophleum suaveolens. Bioresource Technology 75: 171-173.

Pandey, K. K. y Pitman, A. J., 2003. FTIR studies of the changes in wood chemistry following decay by brown-rot and white-rot fungi. International Biodeterioration \& Biodegradation 52: 151-160.

Reid, I. D., 1995. Biodegradation of lignin. Ca. J. Bot. 73 (Suppl. 1): S1011-S1018.

Rodríguez Barreal, J. A., 1998. Patología de la madera. Escuela Técnica Superior de Ingenieros de Montes. Madrid, España. 349 pp.

Roth, I. y Giménez de Bolsón, A. M., 1997. Argentine Chaco forests: Dendrology, tree estructure and economic use. 1. The semi-arid Chaco. Encyclopedia of Plant Anatomy, Berlin, Stuttgart, Borntraeger.184 pp.

Rubilar, O.; Diez, M. C. y Gianfreda, L., 2008. Transformation of chlorinated phenolic compound by white rot fungi. Critical Reviews in Environmental Cience and Technology, 38: 227-268.

Saparrat, M. C. N.; Martínez, M. J.; Cabello, M. N. y Arambarri, A. M., 2002. Screening for ligninolytic enzimes in autochthonous fungal strain from Argentine isolated from different substrata. Rev. Iberoam. Micol. 19: 181-185.

Schmidt, O., 2006. Wood and tree decay. Biology, Damage, Protection and Use. Springer- Verlag Berlin Heidelberg. $329 \mathrm{pp}$.

Schwarze, F. W. M. R., 2007. Wood decay under microscope. Fungal Biology Reviews 21: 133-170.

Schwarze, F. W. M. R; Engels, J. y Mattheck, C., 2000. Fungal strategies of wood decay in trees. Springer Verlag, Berlin. $184 \mathrm{pp}$.

Schwarze, F. W. M. R.; Fink, S. y Deflorio, G., 2003. Resistance of parenchyma cells in wood to degradation by brown rot fungi. Mycological Progress 2(4): 267-274.

Thévenon, M. F.; Tondi, G. y Pizzi, A., 2010. Environmentally friendly wood preservative system based on polymerized tannin resin-boric acid for outdoor applications. Maderas, Ciencia y Tecnología 12(3): 253-257.

Velásquez, J.; Toro, M. E.; Rojas, L. y Lencinas, O., 2006. Actividad antifúngica in vitro de los extractivos naturales de especies latifoliadas de la Guayana Venezolana. Madera y Bosques 12 (1): 51-61.

Wilcox, W., 1978. Review of literature on the effects of early stages of decay on wood strength. Wood and Fiber, $9(4): 252-257$.

Winandy, J. y Morrell, J. J., 1993. Relationship between incipient decay, strength, and chemical composition of Douglas - Fir heartwood. Wood and Fiber Science, 25 (3): 278-288.

Zabel, R. A. y Morrell, J. J., 1992. Wood microbiology. Decay and its prevention. Academics Press Inc. 476 pp. 